\title{
A Wireless Passive SAW Delay Line Temperature and Pressure Sensor for Monitoring Water Distribution System
}

\author{
Zhaozhao Tang ${ }^{1}$, Wenyan $\mathrm{Wu}^{* 2}$, Jinliang Gao ${ }^{3}$ \\ ${ }^{1}$ School of Computing and Digital Technologies, Staffordshire University, Stoke on Trent, United Kingdom \\ ${ }^{2}$ School of Engineering and the Built Environment, Birmingham City University, Birmingham, United Kingdom \\ ${ }^{3}$ School of Municipal and Environmental Engineering, Harbin Institute of Technology, Harbin, P. R. China \\ *wenyan.wu@bcu.ac.uk
}

\begin{abstract}
Wireless passive surface acoustic wave (SAW) sensor has been widely used in many applications. This paper presents a feasibility study on a designed wireless passive SAW delay line temperature and pressure sensor for monitoring water distribution systems. The substrate of the sensor node is a $\mathrm{Y}-\mathrm{Z}$ orientation cut $\mathrm{LiNbO}_{3}$ crystalline with $0.5 \mathrm{~mm}$ thickness. The Interdigital transducer (IDT) was fabricated centrally on the surface of the substrate with an antenna connected. There are three reflectors fabricated on the same surface of the IDT. One reflector is on one side and the other two are on the other side of the IDT. A simulated water pipe platform with testing devices was assembled to test the feasibility of the sensor node working in water pipe environment. The experimental result showed that the designed sensor worked properly but $4 / 5$ amplitude of signals is lost compared to the experimental results in the open-air environment.
\end{abstract}

Keywords-SAW; sensor; temperature; pressure; water distribution

\section{INTRODUCTION}

Wireless passive SAW sensor has been widely used in many industrial applications. It provides the capability of sensing both physical parameters, e.g., temperature [1-5], pressure [4, 5], torsion [6], flow rate [7] etc. and chemical quantities [8-10] without batteries for power supply. They could be activated and powered by the interrogation radio frequency (RF) pulse signals. Compared to conventional active sensors, there are many advantages of using wireless passive SAW sensors apart from their wireless access and passive characters, e.g. low-cost device, reliable element, small size, high sensitivity, and suitable to use in harsh environment [11].

The present work aims to test the feasibility of the designed wireless passive SAW delay line temperature and pressure sensor working in monitoring water distribution systems. The design of the sensor node is introduced in Section II. The experiment set up and test method is presented in Section III. The experimental results are discussed an analysed in Section IV. Section V concludes the findings of this work.

\section{SENSOR NODE DESIGN}

The structure of the wireless passive SAW delay line temperature and pressure sensor node is shown in Figure 1. The substrate is a Y-Z orientation cut $\mathrm{LiNbO}_{3}$ crystalline with 0.5 $\mathrm{mm}$ thickness. The IDT was fabricated in the centre on the surface of the substrate with an antenna connected. Three reflectors were fabricated on the same surface of the IDT. One reflector (R1) is on one side of the IDT for pressure sensing purpose, and the other two (R2 and R3) are on the other side of the IDT for temperature compensation and sensing purpose. The SAW sensor node works relying on the essential energy from the RF pulse signals received by the antenna. In this case, the SAW sensor node contains a minimum number of elements and there are no power supply components, such as batteries, in this design. The ambient pressure applied on the action point is different from the reference pressure inside the sensor. The pressure differences act on the left part of the substrate, and the temperature affects the whole substrate in the meantime by the bonding basis on the right of the substrate. The RF signal can be received and sent through the connected antenna and converted by the IDT. Reflectors are manufactured in parallel with the IDT on the substrate to reflect the SAW. This design can be full use of the SAW energy in two opposite directions propagating from the IDT.

The dimensions of the top surface of the substrate change with the change of temperature and pressure. Consequently, the time delays of the SAW propagations are changed, which leads to the phase shifts in the response signals. In the case of this SAW sensor, the interrogator transmits a pulse signal, and the sensor responds with a chain of pulses depending on the positions of the reflectors arranged on the surface of the substrate. The changes of the time delays of two or more response signals and the original interrogation signal are evaluated. The current pressure and temperature information can be deciphered from the response signals through the subsequent signal processing and analysis. 

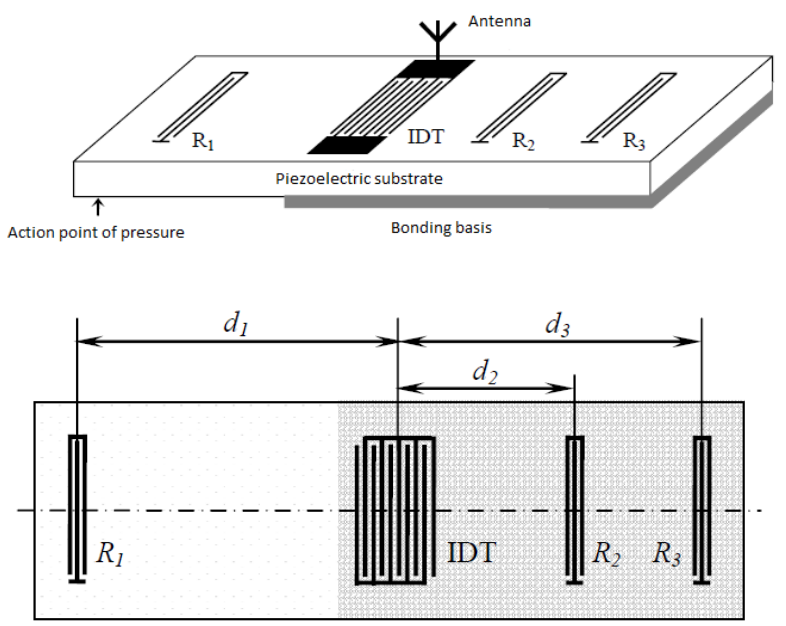

Fig. 1. The structure of the wireless passive SAW temperature and pressure sensor node.

Through theoretical analysis of this design, the relations between the phases and the temperature and pressure values can be described by the following equations,

$$
\begin{gathered}
T-T_{0}=A\left(\varphi_{T}-\varphi_{T_{0}}\right) \\
P-P_{0}=B\left(\varphi_{P T}-\varphi_{P_{0} T_{0}}\right)-C\left(T-T_{0}\right)
\end{gathered}
$$

where $T$ and $T_{0}$ are the temperature value and the initial temperature value respectively. $\varphi_{T}$ is the temperature related response signal phase delay, and $\varphi_{T_{0}}$ is the initial value of $\varphi_{T}$. $A$ is a constant value related to the sensor structure and materials. Similarly, $P$ and $P_{0}$ are the pressure value and the initial pressure value respectively. $\varphi_{P T}$ is the both temperature and pressure related response signal phase delay, and $\varphi_{P_{0} T_{0}}$ is the initial value of $\varphi_{P T} . B$ is a constant value related to the sensor structure and materials. $C$ is a constant only related to materials. Equation (1) and (2) indicate that the related phase delay of the response signal depends linearly on the temperature (pressure) when the pressure (temperature) keeps constant.

\section{EXPERIMENTS}

The experimental platform is shown in Figure 2, the signal generator, the signal analyser and the oscilloscope work as the function of the interrogator for the wireless passive SAW sensor node. Figure 3 is the wireless passive SAW delay line temperature and pressure sensor node with antenna connected to the IDT. The temperature is controlled by the water bath, which is connected to the outer layer of the glass tube. Water with specific temperature circulates between the water bath container and the outer layer of the glass tube. The castor oil pressure control platform is connected to the strictly sealed inner chamber of the glass pipe. Based on the Boyle's Law, the pressure applied via the platform is equal to the air pressure sensed by the sensor node placed in the top of the inner chamber. The Function/Arbitrary Waveform Generator (Agilent 33220A) generates the pulse signal to mix with the standard $425 \mathrm{MHz}$ sine wave generated by the ESG Vector Signal Generator (Agilent E4438C). The multiplication result of the two signals is transmitted out as the interrogation signal to the sensor node. The $1 \mathrm{GHz}$ Mixed Signal Oscilloscope (Agilent MSO6104A) and the Spectrum Analyser (HP 8563E) receive and process both the interrogation signal and the response signals. Based on the previous studies in [4], the centre frequency of these sensor nodes is $425 \mathrm{MHz}$, and the best interrogation pulse width is 1 $\mu \mathrm{s}$. The experiments were undertaken by the above settings.

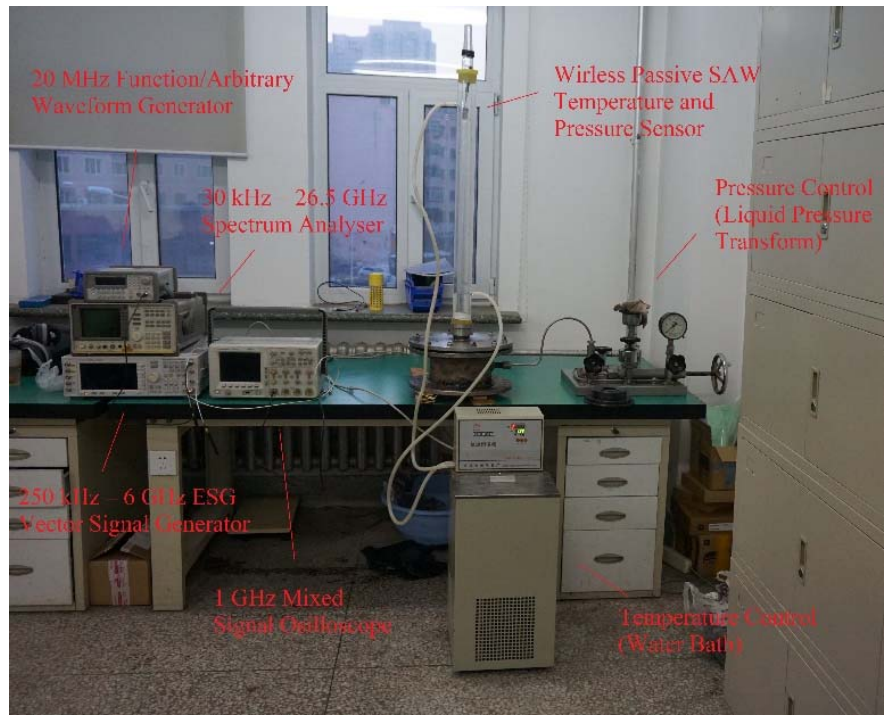

Fig. 2. The picture of the experiment framework set up.

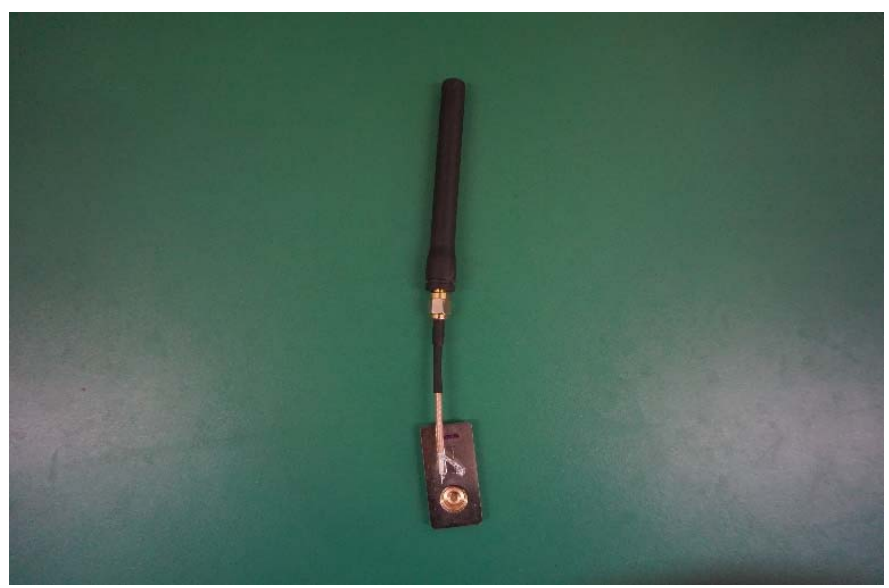

Fig. 3. The wireless passive SAW delay line temperature and pressure sensor node with antenna connected.

The actual temperature and pressure change can be read from the temperature and pressure control platforms, while the experimental data of the interrogation and response signals can be received by the signal analyser and oscilloscope for further processing and analysis.

Firstly, a series of temperature related experimental data are obtained by only changing the temperature through the water bath. Then the pressure related experimental data are obtained by only changing the pressure applied to the inner chamber of the glass pipe. Thirdly, at every selected discrete pressure value point, temperature is changed to record data. 


\section{RESULTS AND DISCUSSIONS}

Wired and wireless interrogation approaches for the sensor nodes were undertaken respectively. The results and analysis are compared and shown in Figure 4(a). The attenuation of the wireless response signals of passive SAW is high against the wired ones. Furthermore, the attenuation increases with the increase of the signal amplitude. There is an average $1 / 5$ attenuation on the response signal when wireless interrogation and response signal receiving process is applied.

The response signals reflected by $R_{2}, R_{3}$ and $R_{1}$ through air and water are compared and analysed. The results are shown in Figure 4(b), 4(c) and 4(d) respectively. These figures indicate that generally the response signals when the passive SAW sensor node is in the experiment container, which is simulating the water pipeline environment just have slight attenuation against the ones when sensor node is in the air. Therefore, the attenuation caused by water could be ignored in the practical engineering environment.

Based on theoretical analysis for this designed wireless passive SAW delay line temperature and pressure sensor in Equation (1) and (2), the related phase delays of the response signals depend linearly on the temperature (pressure) when the pressure (temperature) keeps constant. The experimental results are shown in Figure 5(a) and 5(b). From these two figures, the experimental results show similar relations as the theoretical analysis, which demonstrates temperature and pressure values can be estimated by the phase delays.

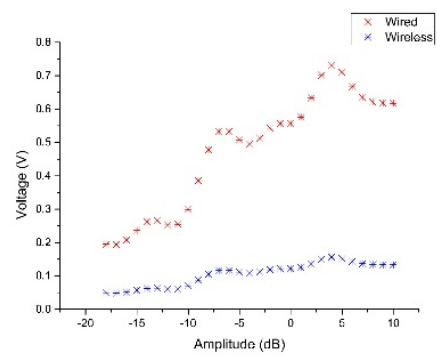

(a)

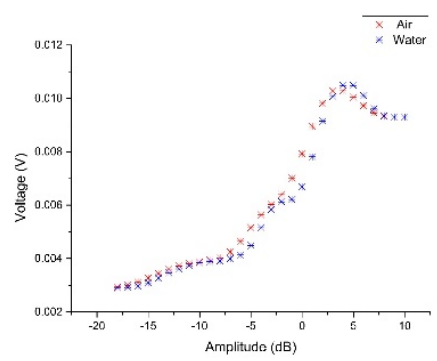

(c)

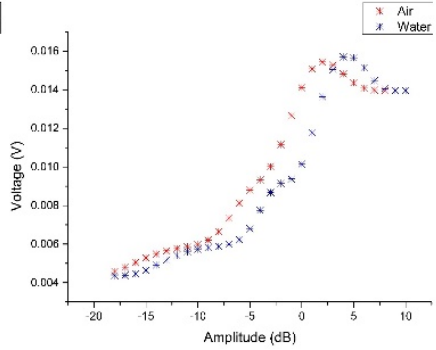

(b)

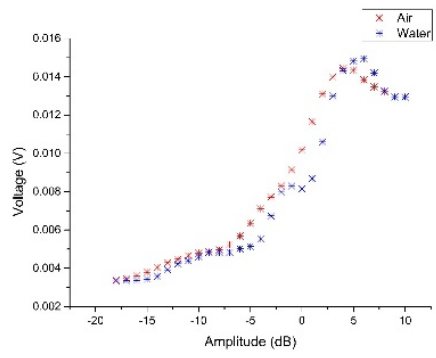

(d)
Fig. 4. (a) The attenuation of the received wireless signal against the wired signal. (b) The comparison of the response signals reflected by $R_{2}$ when the SAW sensor node was put in the air and in the simulating water pipeline condition. (c) The comparison of the response signals reflected by $\mathrm{R}_{3}$ when the SAW sensor node was put in the air and in the simulating water pipeline condition. (d) The comparison of the response signals reflected by $\mathrm{R}_{1}$ when the SAW sensor node was put in the air and in the simulating water pipeline condition.

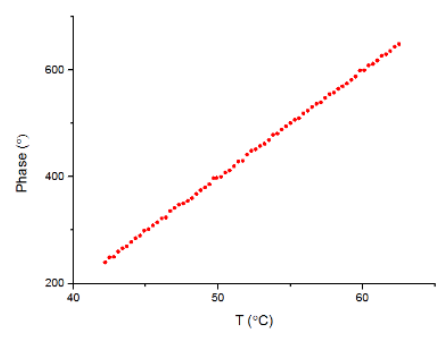

(a)

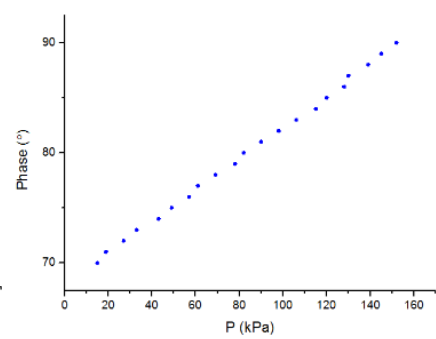

(b)
Fig. 5. (a) The relation between the phase delay and temperature when the pressure keeps constant. (b) The relation between the phase delay and pressure when the temperature keeps constant.

\section{CONCLUSIONS}

A wireless passive SAW temperature and pressure delay line sensor was adapted in a designed experimental framework which can simulate the temperature and pressure changes in the water distribution pipelines. The amplitudes of the wired and wireless response signals are compared. 4/5 amplitude is lost on the response signals when wireless interrogation and response signal receiving process is applied. The response signals of the sensor node in different ambient environments are analysed and compared. Compared to the open-air environment, there is only slight attenuation of the response signals when the sensor node is planted in the designed experimental framework. The related phase delays of the response signals depend linearly on the temperature when the pressure keeps constant, and the related phase delays of the response signals depend linearly on the pressure when the temperature keeps constant. The experimental results meet the theoretical relations well. Therefore, this designed wireless passive SAW delay line temperature and pressure sensor is feasible to work in the water pipe environment.

\section{ACKNOWLEDGMENT}

The authors acknowledge the financial support of the European Commission $7^{\text {th }}$ Framework Programme (EU FP7) WatERP (318603), EU FP7 Marie Curie Actions - SmartWater (318985) and EU Horizon 2020 Marie Curie Actions - ITN IOT4win (765921) and the support of Dr.Tianli Li from Shenzhen University, China.

\section{REFERENCES}

[1] G. Bruckner, J. Bardong, C. Gruber, and V. Plessky, "A wireless, passive ID tag and temperature sensor for a wide range of operation," Procedia Engineering, vol. 47, pp. 132-135, 2012.

[2] A. Kang, C. Zhang, X. Ji, T. Han, R. Li, and X. Li, "SAW-RFID enabled temperature sensor," Sensors and Actuators: A, Physics, vol. 201, pp. 105-113, 2013.

[3] C. Fu, Y. Ke, M. Li, J. Luo, H. Li, G. Liang, and P. Fan, "Design and Implementation of $2.45 \mathrm{GHz}$ Passive SAW Temperature Sensors with BPSK Coded RFID Configuration," Sensors, vol. 17, no. 8, pp. 1849, Aug. 2017.

[4] Z. Tang, W. Wu, and J. Gao, "Water pressure sensing based on wireless passive SAW technology," 13th International Conference on Computing and Control for the Water Industry, CCWI2015, Procedia Engineering, vol.119, pp. 892-900, 2015.

[5] Z. Tang, W. Wu, J. Gao and P. Yang, "Feasibility Study on Wireless Passive SAW Sensor in IoT Enabled Water Distribution System," In Internet of Things (iThings) and IEEE Green Computing and 
Communications (GreenCom) and IEEE Cyber, Physical and Social Computing (CPSCom) and IEEE Smart Data (SmartData), 2017 IEEE International Conference on, pp. 830-834, 2017.

[6] C. Lin, C. Hung, and H. Lin, "A study of wireless torque sensing based on SAW sensors," 2010 International Symposium on Computer, Communication, Control and Automation (3CA), vol. 2, pp. 211-214, 2010.

[7] Y. Wang, Z. Li, L. Qin, M. Chyu, and Q. Wang, "Theoretical and experiemntal studies of a surface acoustic wave flow sensor," IEEE Transactions on Ultrasonics, Ferroelectrics, and Frequency Control, vol. 59, No. 3, pp. 481-490, 2012.
[8] R. Yadava and V. Verma, "A diffusion limited sorption-desorption noise model for polymer coated SAW chemical sensors," Sensors and Actuators B: Chemical, vol. 195, pp. 590-602, 2014.

[9] L. Rana, R. Gupta, A. Sharma, V. Gupta and M. Tomar, "Novel designs of SAW devices for highly sensitive chemical sensors," Materials Today: Proceedings, vol 5, issue 7, part 2, pp. 15371-15375, 2018.

[10] R. Kshetrimayum, "Effects of the set phase position on the response of a SAW resonator oscillator based chemical sensor," IEEE Sensors Journal, vol. 13, issue 5, pp. 1662-1668, 2013.

[11] D. C. Malocha, M. Gallagher, B. Fisher, J. Humphries, D. Gallagher and N. Kozlovski, "A Passive Wireless Multi-Sensor SAW Technology Device and System Perspectives," Sensors, vol. 13, pp. 5897-5922, 2013. 